\title{
Linear Confinement of a Relativistic Spin-0 scalar Particle under Lorentz Symmetry Violation Effects
}

\author{
Faizuddin Ahmed ${ }^{1}$ \\ National Academy Gauripur, Assam, 783331, India
}

\begin{abstract}
In this work, linear confinement of a relativistic scalar particle under the effects of Lorentz symmetry violation is investigated. We introduce a scalar potential by modifying the mass via transformation $M \rightarrow M+S(r)$ in the wave equation, and analyze the effects on the eigenvalues and the wave function. We see that the solution of the bound state to the wave equation can be achieved, and the energy eigenvalues and the wave function modified by the Lorentz symmetry breaking parameters as well as potential.
\end{abstract}

Keywords: Lorentz symmetry violation, Relativistic wave-equations, electric \& magnetic field, special function.

PACS Number(s): 03.65.Pm, 11.30.Cp, 11.30.Qc

\section{Introduction}

The development of Lorentz symmetry breaking (LSV) in quantum field theory has allowed the formulation of a new model that aims to go beyond the Standard Model which is known as Standard Model Extension (SME) $[1,2,3,4]$. The gauge sector of the SME are classified into two sector called the CPT-odd sector $[1,2]$ and the CPT-even sector $[5,6,7,8,9,10,11]$. It is worth mentioning that the SME keeps the gauge invariance, the conservation of energy and momentum, and the covariance under observer rotations and

\footnotetext{
${ }^{1}$ faizuddinahmed15@gmail.com ; faiz4U.enter@rediffmail.com
} 
boosts. Recently, by nonminimal coupling the CPT-even sector of the SME has been applied in the analysis of the quantum dynamics of a scalar field under the effects of central potentials induced by the LSV, for example, in the nonrelativistic limit Refs. $[12,13,14,15,16,17,18,19,20,21,22,23]$; In the relativistic limit Refs. [24, 25, 26, 27, 28, 29, 30, 31, 32, 33, 34, 35].

For the studies of spin-0 scalar particles under the LSV effects, the background of Lorentz symmetry violation is introduced into the wave equation through a nonminimal coupling given by $\frac{g}{4}\left(K_{F}\right)_{\mu \nu \alpha \beta} F^{\mu \nu}(x) F^{\alpha \beta}(x)$, where $g$ is the coupling constant, $F_{\mu \nu}(x)$ is the electromagnetic field tensor, and $\left(K_{F}\right)_{\mu \nu \alpha \beta}$ is a dimensionless tensor that governs LSV out of the SME. The tensor $\left(K_{F}\right)_{\mu \nu \alpha \beta}$ has the symmetries of the Riemann tensor $R_{\mu \nu \alpha \beta}$ and zero on double trace $\left(K_{F}\right)_{\mu \nu}^{\mu \nu}=0$ (for details of the tensor $\left(K_{F}\right)_{\mu \nu \alpha \beta}$, see Refs. $[1,2,7,8,9,10,11])$. Therefore, the wave equation of scalar particles under the effects of LSV Refs. [1, 2, 19, 25, 26, 27, 28, 29, 30, 31] is given by

$$
p^{\mu} p_{\mu} \Psi+\frac{g}{4}\left(K_{F}\right)_{\mu \nu \alpha \beta} F^{\mu \nu}(x) F^{\alpha \beta}(x) \Psi=M^{2} \Psi,
$$

where $\Psi$ is the scalar wave function, and $M$ is the rest mass of the particle.

If one introduce a scalar potential in the quantum systems, the known procedure was given in Refs. [36, 37, 38], where the mass term $M$ is modified via transformation $M \rightarrow M+S(r)$, where $S(r)$ is a scalar potential. The scalar potentials of various kind have been used to obtain the solution of the bound state to the relativistic wave equations under LSV effects Refs. $[25,27,28,29,30]$. In this work, we consider a linear confining potential proportional to the axial distance $(\propto r)$ [30, 38, 39], then, the mass term of the KleinGordon equation becomes

$$
M \rightarrow\left(M+\eta_{L} r\right)
$$

where $\eta_{L}$ is a constant that characterizes the linear conning potential.

We investigate the relativistic quantum motion of scalar particles under the Lorentz symmetry breaking effects defined by a tensor $\left(K_{F}\right)_{\mu \nu \alpha \beta}$ out of 
the Standard Model Extension (SME) in the presence of a linear confining potential which hasn't yet been investigated.

The structure of this paper is as follows: in section 2, we introduce the Lorentz symmetry violation defined by a tensor $\left(K_{F}\right)_{\mu \nu \alpha \beta}$ out of the Standard Model Extension. Then, we analyze the behaviour of a relativistic scalar particle in the presence of a linear confining potential by solving the KleinGordon equation under the effects of the Lorentz symmetry violation; in section 3, we present our conclusions.

\section{Lorentz Symmetry Breaking Effects on a Relativistic Scalar Particle with potential}

We consider the Minkowski flat space-time in the cylindrical coordinates $(t, r, \phi, z)$

$$
d s^{2}=-d t^{2}+d r^{2}+r^{2} d \phi^{2}+d z^{2}
$$

where the ranges of the coordinates are $-\infty<(t, z)<\infty, r \geq 0$ and $0 \leq \phi \leq 2 \pi$.

For the geometry (3), the modified Klein-Gordon equation (1) in the presence of a linear potential defined by (2) becomes

$$
\begin{aligned}
& {\left[-\frac{\partial^{2}}{\partial t^{2}}+\frac{\partial^{2}}{\partial r^{2}}+\frac{1}{r} \frac{\partial}{\partial r}+\frac{\partial^{2}}{\partial z^{2}}+\frac{1}{r^{2}} \frac{\partial^{2}}{\partial \phi^{2}}\right] \Psi+\frac{g}{4}\left(K_{F}\right)_{\mu \nu \alpha \beta} F^{\mu \nu}(x) F^{\alpha \beta}(x) \Psi} \\
& =\left(M+\eta_{L} r\right)^{2} \Psi
\end{aligned}
$$

Now using the properties of the tensor $\left(K_{F}\right)_{\mu \nu \alpha \beta}$ given in Refs. $[7,8,9$, $25,27,28,29,30]$, we can rewrite (4) in the following form :

$$
\begin{aligned}
& {\left[-\frac{\partial^{2}}{\partial t^{2}}+\frac{\partial^{2}}{\partial r^{2}}+\frac{1}{r} \frac{\partial}{\partial r}+\frac{1}{r^{2}} \frac{\partial^{2}}{\partial \phi^{2}}+\frac{\partial^{2}}{\partial z^{2}}\right] \Psi} \\
& +\left[-\frac{g}{2}\left(\kappa_{D E}\right)_{i j} E^{i} E^{j}+\frac{g}{2}\left(\kappa_{H B}\right)_{j k} B^{i} B^{j}-g\left(\kappa_{D B}\right)_{j k} E^{i} B^{j}\right] \Psi \\
& =\left(M+\eta_{L} r\right)^{2} \Psi
\end{aligned}
$$


Let us consider a possible scenario of the Lorentz symmetry violation determined by $\left(\kappa_{D E}\right)_{11}=$ const, $\left(\kappa_{H B}\right)_{33}=$ const, $\left(\kappa_{D B}\right)_{13}=$ const and the field configuration given by $[16,27,30,31,34,35]$ :

$$
\vec{B}=B_{0} \hat{z} \quad, \quad \vec{E}=\frac{\lambda}{r} \hat{r}
$$

where $B_{0}>0, \hat{z}$ is a unit vector in the $z$-direction, $\lambda$ is the linear charge density, and $\hat{r}$ is the unit vector in the radial direction.

Hence, equation (5) using the field configuration (6) becomes

$$
\begin{aligned}
& {\left[-\frac{\partial^{2}}{\partial t^{2}}+\frac{\partial^{2}}{\partial r^{2}}+\frac{1}{r} \frac{\partial}{\partial r}+\frac{1}{r^{2}} \frac{\partial^{2}}{\partial \phi^{2}}+\frac{\partial^{2}}{\partial z^{2}}\right] \Psi} \\
& +\left[-\frac{g}{2}\left(\kappa_{D E}\right)_{11} \frac{\lambda^{2}}{r^{2}}+\frac{g}{2}\left(\kappa_{H B}\right)_{33} B_{0}^{2}-\frac{g \lambda B_{0}}{r}\left(\kappa_{D B}\right)_{13}\right] \Psi=\left(M+\eta_{L} r\right)^{2} \Psi .
\end{aligned}
$$

Since the metric (3) is independent of $(t, \phi, z)$, let us choose the possible total wave function by

$$
\Psi(t, r, \phi, z)=e^{i(-\varepsilon t+l \phi+k z)} \psi(r)
$$

where $\varepsilon$ is the energy of the particle, $l=0, \pm 1, \pm 2, \ldots$ are the eigenvalues of the $z$-component of the angular momentum operator, and $k$ is the constant.

Substituting the solution (8) into the Eq. (7), we obtain the following radial wave-equation for $\psi(r)$ :

$$
\psi^{\prime \prime}(r)+\frac{1}{r} \psi^{\prime}(r)+\left[\Lambda-\eta_{L}^{2} r^{2}-\frac{j^{2}}{r^{2}}-\frac{\delta}{r}-\beta r\right] \psi(r)=0,
$$

where

$$
\begin{aligned}
\Lambda & =\varepsilon^{2}+\frac{1}{2} g B_{0}^{2}\left(\kappa_{H B}\right)_{33}-M^{2}-k^{2}, \\
j & =\sqrt{l^{2}+\frac{1}{2} g \lambda^{2}\left(\kappa_{D E}\right)_{11}}, \\
\delta & =g \lambda B_{0}\left(\kappa_{D B}\right)_{13}, \\
\beta & =2 M \eta_{L} .
\end{aligned}
$$


Let us now perform a change of variable by $x=\sqrt{\eta_{L}} r$. Then, from Eq. (9) we have

$$
\psi^{\prime \prime}(x)+\frac{1}{x} \psi^{\prime}(x)+\left[\Delta-x^{2}-\frac{j^{2}}{x^{2}}-\frac{\xi}{x}-\chi x\right] \psi(x)=0,
$$

where

$$
\Delta=\frac{\Lambda}{\eta_{L}} \quad, \quad \xi=\frac{\delta}{\sqrt{\eta_{L}}} \quad, \quad \chi=\frac{\beta}{\eta_{L}^{\frac{3}{2}}}
$$

By analysing the asymptotic behaviour of the Eq. (11) at $x \rightarrow 0$ and at $x \rightarrow \infty$, we have a solution to the Eq. (11) that can be written in terms of an unknown function $F(x)$ as

$$
\psi(x)=x^{j} e^{-\frac{1}{2}(x+\chi) x} H(x)
$$

Thereby, substituting the solution (13) into the Eq. (11), we obtain the following equation:

$$
H^{\prime \prime}(x)+\left[\frac{1+2 j}{x}-2 x-\chi\right] H^{\prime}(x)+\left[-\frac{\beta}{x}+\Pi\right] H(x)=0,
$$

where

$$
\Pi=\Delta+\frac{\chi^{2}}{4}-2(1+j) \quad, \quad \beta=\xi+\frac{\chi}{2}(1+2 j)
$$

Equation (14) is the biconfluent Heuns dierential equation [38, 25, 27, 28, 29, 30] with $H(x)$ is the Heun function.

The above equation (14) can be solved by the Frobenius method. Writing the solution as a power series expansion around the origin [40]:

$$
H(x)=\sum_{i=0}^{\infty} d_{i} x^{i}
$$

Substituting the power series solution into the Eq. (14), we obtain the following recurrence relation

$$
d_{n+2}=\frac{1}{(n+2)(n+2+2 j)}\left[\left\{\xi+\chi\left(n+j+\frac{3}{2}\right)\right\} d_{n+1}-(\Pi-2 n) d_{n}\right]
$$


With the few coefficients

$$
d_{1}=\left(\frac{\xi}{1+2 j}+\frac{\chi}{2}\right) d_{0} \quad, \quad d_{2}=\frac{1}{4(1+j)}\left[\left\{\xi+\chi\left(j+\frac{3}{2}\right)\right\} d_{1}-\Pi d_{0}\right]
$$

The power series expansion $H(x)$ becomes a polynomial of degree $\mathrm{n}$ by imposing the following two conditions [38, 25, 27, 28, 29, 30]

$$
\Pi=2 n(n=1,2,3, \ldots) \quad, \quad d_{n+1}=0 .
$$

Analyzing the first condition $\Pi=2 n$, we have obtained the following equation of the energy $\varepsilon_{n, l}$ :

$$
\varepsilon_{n, l}= \pm \sqrt{k^{2}+2 \eta_{L}\left(n+1+\sqrt{l^{2}+\frac{1}{2} g \lambda^{2}\left(\kappa_{D E}\right)_{11}}\right)-\frac{g}{2} B_{0}^{2}\left(\kappa_{H B}\right)_{33}}
$$

The radial wave function is given by

$$
\psi_{n, l}(x)=x^{\sqrt{l^{2}+\frac{1}{2} g \lambda^{2}\left(\kappa_{D E}\right)_{11}}} e^{-\frac{1}{2}\left[x+\frac{M}{\sqrt{\eta_{L}}}\right] x} H(x) .
$$

Now, we evaluate the individual energy levels and eigenfunctions one by one as done in Refs. [38, 25, 27, 28, 29, 30]. For example, $n=1$, we have $\Pi=2$ and $d_{2}=0$ which implies

$$
\begin{aligned}
& \frac{2}{\xi+\frac{\chi}{2}\left(j+\frac{3}{2}\right)} d_{0}=\left(\frac{\xi}{1+2 j}+\frac{\chi}{2}\right) d_{0} \\
& \Rightarrow \eta_{L}^{1, l}=\left[\frac{\left\{\frac{\delta}{2}+M\left(\frac{3}{2}+j\right)\right\}\left\{\frac{\delta}{2}+M\left(\frac{1}{2}+j\right)\right\}}{\left(\frac{1}{2}+j\right)}\right]
\end{aligned}
$$

a constraint on the potential parameter $\eta_{L}^{1, l}$. We can see, from Eq. (23) that the allowed values of the potential parameter depends on quantum numbers $\{n, l\}$ of the system and the Lorentz symmetry breaking parameter $\left\{g, B_{0}, \lambda,\left(\kappa_{D B}\right)_{13},\left(\kappa_{D E}\right)_{11}\right\}$. Similarly, one can find another relation of this potential parameter $\eta_{L}^{2, l}$ for the radial mode $n=2$ and so on. 
Therefore, the ground state energy level for the radial mode $n=1$ is given by

$$
\varepsilon_{1, l}= \pm \sqrt{k^{2}+2 \eta_{L}^{1, l}\left(2+\sqrt{l^{2}+\frac{1}{2} g \lambda^{2}\left(\kappa_{D E}\right)_{11}}\right)-\frac{1}{2} g B_{0}^{2}\left(\kappa_{H B}\right)_{33}} .
$$

And the ground state wave function is

$$
\psi_{1, l}(x)=x^{\sqrt{l^{2}+\frac{1}{2} g \lambda^{2}\left(\kappa_{D E}\right)_{11}}} e^{-\frac{1}{2}\left[x+\frac{2 M}{\sqrt{\eta_{L}^{1, l}}}\right] x}\left(1+d_{1} x\right),
$$

where

$$
d_{1}=\frac{1}{\sqrt{\eta_{L}^{1, l}}}\left[\frac{g \lambda B_{0}\left(\kappa_{D B}\right)_{13}}{1+2 \sqrt{l^{2}+\frac{1}{2} g \lambda^{2}\left(\kappa_{D E}\right)_{11}}}+M\right]
$$

Equation (24) gives the lowest state energy level, Eqs. (25)-(26) is the round state wave function with the restriction (23) on the potential parameter under the effects Lorentz symmetry violation for the radial mode $n=1$. The spectrum of energy Eq. (24) is symmetrical about $\varepsilon_{n}=0$ for constant or zero values of $l$ and are equally spaced on either side. We have established the background of the violation of the Lorentz symmetry defined by a tensor possessing the non-null components $\left\{\left(\kappa_{D E}\right)_{11}=\right.$ const, $\left(\kappa_{H B}\right)_{33}=$ const, $\left(\kappa_{D B}\right)_{13}=$ const $\}$.

\section{Conclusions}

We have investigated the effects of Lorentz symmetry violation (LSV) on a relativistic scalar particle by solving the Klein-Gordon equation in the presence of a linear confining potential. Though our work is inspired by the Standard Model Extension (SME) that is an effective field theory, we have relaxed the renormalization property in our analysis. After solving the radial wave equation, we have obtained the energy spectrum Eq. (20), and the wave function Eq. (21) of a relativistic scalar particle. It is worth mentioning that Eq. (20) is not the general expression of the energy eigenvalues. 
One can obtain the individual energy level and wave function one by one by imposing the recurrence condition $d_{n+1}=0$. In this work, we have one such energy level (24), the ground state wave function (25)-(26) with the restriction (23) on the potential parameter for the radial mode $n=1$ and others are in the same way. We have shown that the solution of the bound state to the Klein-Gordon equation can be obtained under the effects of the Lorentz symmetry violation defined by a radial electric field produced by linear distribution of electric charge, a uniform magnetic field along the $z$-direction, and the background dimensionless tensor $\left(K_{F}\right)_{\alpha \beta \mu \nu}$ that governs the Lorentz symmetry breaking possessing the non-null components $\left(\kappa_{D B}\right)_{13}=$ const, $\left(\kappa_{D E}\right)_{11}=$ const and $\left(\kappa_{H B}\right)_{33}=$ const. We can see that the presence of the above non-null components of the tensor $\left(K_{F}\right)_{\mu \nu \alpha \beta}$ and the linear confining potential modified the spectrum of energy Eq. (20) and the wave function (21).

\section{Conflict of Interest}

There is no conflict of interest regarding publication this paper.

\section{Data Availability}

No data has been used to prepare this manuscript.

\section{References}

[1] D. Colladay and V. A. Kosteleck, Phys. Rev. D 55, 6760 (1997).

[2] D. Colladay and V. A. Kostelecky, Phys. Rev. D 58, 116002 (1998).

[3] V. A. Kostelecky and R. Potting, Phys. Rev. D 51, 3923 (1995).

[4] V. A. Kostelecky and R. Potting, Phys. Lett. B 381, 89 (1996). 
[5] S. Carroll, G. Field and R. Jackiw, Phys. Rev. D 41, 1231 (1990).

[6] A. P. B. Scarpelli, H. Belich, J. L. Boldo, L. P. Colatto, J. A. HelayelNeto and A. L. M. A. Nogueira, Nucl. Phys. (Proc. Suppl.) 127, 105 (2004).

[7] V. A. Kostelecky and M. Mewes, Phys. Rev. Lett. 87, 251304 (2001).

[8] V. A. Kostelecky and M. Mewes, Phys. Rev. D 66, 056005 (2002).

[9] V. A. Kostelecky and M. Mewes, Phys. Rev. Lett. 97, 140401 (2006).

[10] H. Belich, F. J. L. Leal, H. L. C. Louzada and M. T. D. Orlando, Phys. Rev. D 86, 125037 (2012).

[11] Quentin G. Bailey and V. Alan Kostelecky, Phys. Rev. D 70, 076006 (2004).

[12] M. M. Ferreira Jr. and F. M. O. Moucherek, Nucl. Phys. A 790, 635 (2007).

[13] L. R. Ribeiro, E. Passos and C. Furtado, J. Phys. G : Nucl. Phys. 39, 105004 (2012).

[14] H. Belich, E. O. Silva, M. M. Ferreira Jr., M. T. D. Orlando, Phys. Rev. D 83, 125025 (2011).

[15] K. Bakke and H. Belich, J. Phys. G : Nucl. Part. Phys. 39, 085001 (2012).

[16] K. Bakke, E. O. Silva and H. Belich, J. Phys. G : Nucl. Part. Phys. 39, 055004 (2012).

[17] K. Bakke and H. Belich, Ann. Phys. (N. Y.) 354, 1 (2015).

[18] R. Casana, M. M. Ferreira Jr., E. Passos, F. E. P. dos Santos and E. O. Silva, Phys. Rev. D 87, 047701 (2013). 
[19] K. Bakke and H. Belich, Ann. Phys. (Berlin) 526, 187 (2014).

[20] K. Bakke and H. Belich, J. Phys. G : Nucl. Part. Phys. 42, 095001 (2015).

[21] K. Bakke and H. Belich, J. Phys. G : Nucl. Part. Phys. 40, 065002 (2013).

[22] A. G. de Lima, H. Belich and K. Bakke, Eur. Phys. J. Plus 128, 154 (2013).

[23] K. Bakke and H. Belich, Eur. Phys. J. Plus 127, 102 (2012).

[24] K. Bakke and H. Belich, Ann. Phys. (N. Y.) 373, 115 (2016).

[25] R. L. L. Vitoria, H. Belich and K. Bakke, Adv. High Energy Phys. 2017, 6893084 (2017).

[26] R. L. L. Vitoria, K. Bakke and H. Belich, Ann. Phys. (N. Y.) 399, 117 (2018).

[27] K. Bakke and H. Belich, Ann. Phys. (N. Y.) 360, 596 (2015).

[28] R. L. L. Vitoria and H. Belich, Eur. Phys. J. C 78, 999 (2018).

[29] K. Bakke and H. Belich, Ann. Phys. (N. Y.) 333, 272 (2013).

[30] R. L. L. Vitoria, H. Belich and K. Bakke, Eur. Phys. J. Plus 132, 25 (2017).

[31] R. L. L. Vitoria and H. Belich, Eur. Phys. J. Plus 135, 247 (2020).

[32] K. Bakke and H. Belich, Int. J. Mod. Phys. A 30, 1550197 (2015).

[33] A. G. de Lima, H. Belich and K. Bakke, Ann. Phys. (Berlin) 526, 514 (2014). 
[34] F. Ahmed, DOI: 10.20944/preprints202105.0748.v1.

[35] F. Ahmed, DOI: 10.20944/preprints202105.0755.v1.

[36] H. G. Dosch, J. H. Jansen, V. F. Mller, Phys. Norv. 5, 2 (1971).

[37] W. Greiner, Relativistic Quantum Mechanics: Wave Equations, 3rd edition (Springer, Berlin, 2000).

[38] E. R. F. Medeiros and E. R. Bezerra de Mello, Eur. Phys. J. C 72, 2051 (2012).

[39] F. Ahmed, Eur. Phys. J. C 78, 598 (2018).

[40] G. B. Arfken and H. J. Weber, Mathematical Methods For Physicists, Elsevier Academic Press, London (2005). 